\title{
Guest Editor: Vipin Kumar Chaturvedi
}

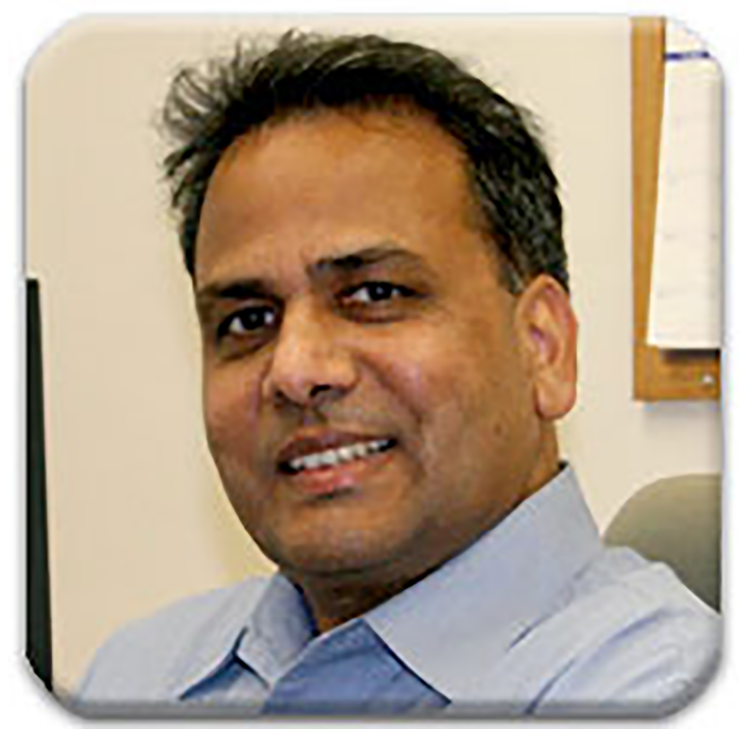

Dr. Vipin Kumar, MS, PhD, Editor-in-Chief of Critical Reviews ${ }^{\mathrm{TM}}$ in Immunology, is currently a Professor in the Department of Medicine at the University of California, San Diego (UCSD). He is also member of the NAFLD Center, the Center for Microbiome Innovation, the Moores Cancer Center, and the Clinical and Translational Research Institute, UCSD and the Southern California Center for
Alcoholic Liver \& Pancreatic Diseases and Cirrhosis at the USC School of Medicine, Los Angeles. His laboratory has made profound contributions related to how autoimmune diseases, such as multiple sclerosis and lupus, are initiated and how they are controlled by different populations of $\mathrm{CD}^{+}$and $\mathrm{CD}^{+}$regulatory $\mathrm{T}$ cells. Some of his work has led to ongoing clinical studies in humans to identify and generate novel therapeutics. Recently, Dr. Kumar's laboratory has also identified different innate-like $\mathrm{T}$ cell populations, including NKT cells subsets, and have characterized their self-lipid antigen recognition and their role in inflammatory diseases, including alcoholic liver disease (ALD) and nonalcoholic steatohepatitis (NASH). Dr. Kumar and his team have also discovered a novel population of innate-like regulatory $\mathrm{CD} 8^{+} \mathrm{T}$ cell population that controls autoimmune and inflammatory pathologies in different organs, including liver and the gut, with translational implications for new treatment strategies. He has several patents and cofounded biotech companies that are involved in clinical studies in humans based upon novel research from his laboratory, and he has been on their scientific advisory boards. He has been publishing his work in high impact journals and is currently Associate Editor for Critical Reviews ${ }^{\mathrm{TM}}$ in Oncogenesis. 